\title{
Case Study on Environmental Impact of Cloud Computing
}

\author{
Rajender Kumar Trivedi, Rajani Sharma \\ Department of Computer Science and Information Technology, Graphic Era University, India \\ Department of Computer Science and Information Technology, Graphic Era University, India
}

\begin{abstract}
With ever increasing usage of IT infrastructure around the globe, the increase in energy consumption and carbon emission is a worrisome situation for everyone because of its direct effect on environment. The environment is being dangerously impacted by Information Technology infrastructure extensive use and its waste. Cloud computing has emerged as one step further solution to deploy all virtualized IT resources as per need on self-service basis as a rental method for the users. Different cloud deployment methods have emerged according to the need of users, institutions and communities. In last decade virtualization has been a milestone in enabling optimal usage of information technology resources. Cloud computing has provided a platform for deploying everything as a Service in IT, which has changed the economics of IT based business and organizations from Capital Expenditure to Operational Expenditure. For enabling Cloud services we first need to understand our existing infrastructure. In second step we should be able to virtualize it for optimal usage. Then with the help of existing cloud applications we can deliver and deploy information technology as a resource at various levels such as infrastructure, platform, and software.
\end{abstract}

Keywords; cloud computing, carbon emission, energy consumption, IT waste, virtualization.

\section{INTRODUCTION}

The Advancement in the field of information technology has enabled us to optimize the use of physical infrastructure with the help of virtualization, most of which is only used in specific applications like vitalizing a local area network on switch or vitalizing a compute resource to run more than one operating system on a desktop. The benefits of same has not been completely implemented and utilized in industrial environments where we require a large amount of IT resources. The advantage of using cloud is to enable complete utilization of Information technology resources, where most of the resources are underutilized. The cost involved in maintenance and management of those resources is huge which can be saved due to centralized management of private cloud infrastructure. We create private cloud keeping in mind.

- Availability and performance requirements for the cloud

- Infrastructure sizing, performance and continual availability requirements

- The need for data security or isolation

- The appropriate user interface

With advent of IT as a service in cloud computing technology energy consumption and related carbon emissions and waste are considerably lesser than in-house implementation. Technology in Cloud Computing creates an able structure for sharing resources with the help of virtualization among many; it greatly decreases or abolishes the need for most products, equipment, utilities and facilities. The results are more sustainable IT infrastructures and processes which help in keeping environment safe.

- Highest resource consumption and effectiveness

- Reduced energy consumption by up to $95 \%$ with the help of virtualization which optimizes the use of physical infrastructure and thereby helps IT to be delivered as a service.

- Displaced utility costs Workstation utility costs are cut by $90 \%$ due to change of capital expenditure into operational expenditure in industries or organizations across the world.

- Reduced electronic waste as No more batteries and minimal software/hardware waste.

- Reduced waste overall due to consumption of computational power as service, no more as infrastructure.

- Reduced ecological impact of workstations as compared to thin clients as anything related to computation can be provided as a service on a client connected with internet to the cloud provider. 


\section{UNDERSTANDing THE CLOUD}

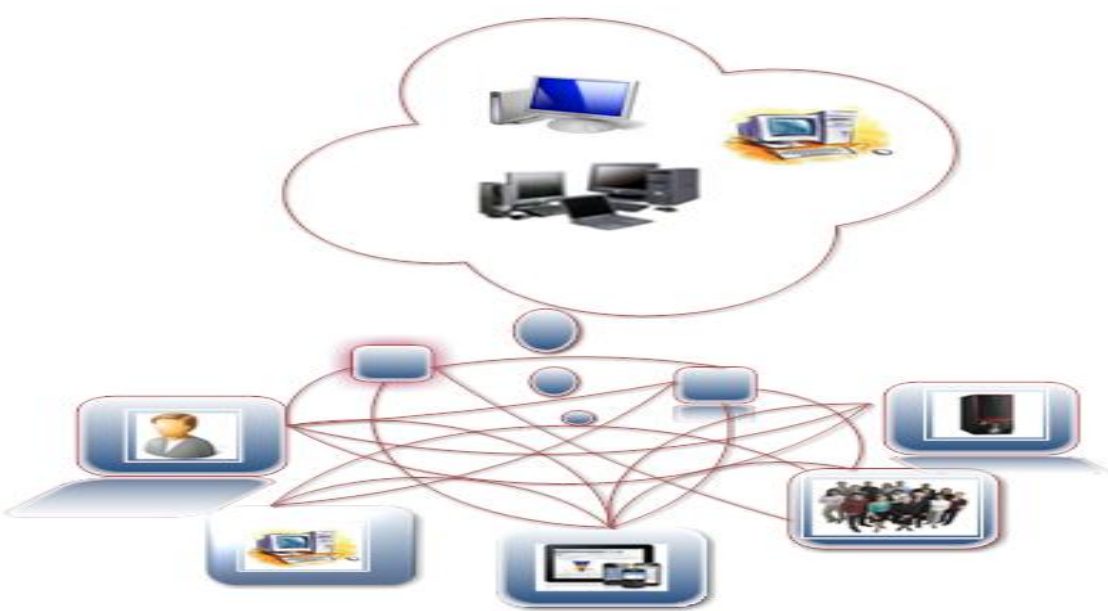

There are majorly three cloud service models existing. Infrastructure as a Service provides capability to the consumer to hire infrastructure components such as servers, storage, and network. It enables consumers to deploy and run software, including OS and applications. Consumer pays for infrastructure components usage, for example, storage capacity, CPU usage, etc.

\section{Cloud---Infrastructure-as-a-service}

\begin{tabular}{|c|} 
APPLICATION \\
DATABASES \\
OS \\
COMPUTE \\
STORAGE \\
NETWORK
\end{tabular}

HIRED RESOURCES

In platform as a Service consumer can deploy consumer-created or acquired applications on the cloud provider's infrastructure. Consumer has control over deployed applications and possible application hosting environment configurations. Consumer is billed for platform software components which include its associated infrastructure cost such as OS, Databases, and Middleware.

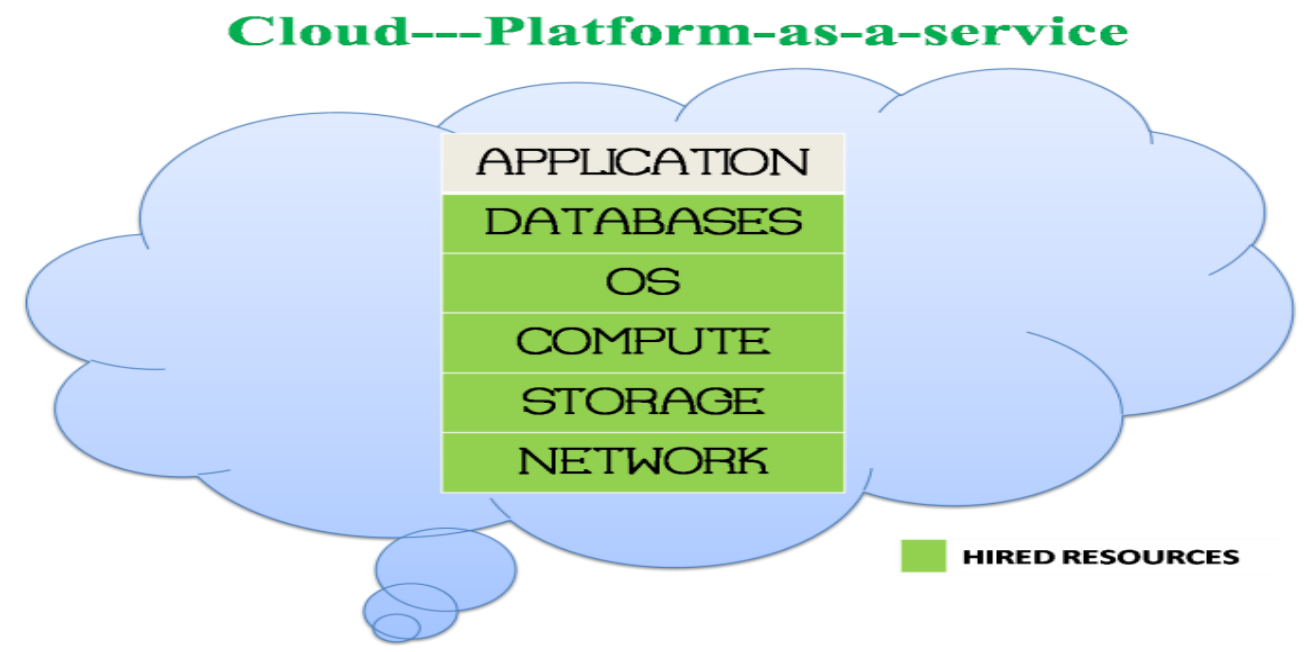

In Software as a Service cloud application consumer can use provider's applications running in a cloud infrastructure. In this complete stack including application is provided as a service. Application is accessible from various client devices, for example, via a thin client interface such as a web browser. 


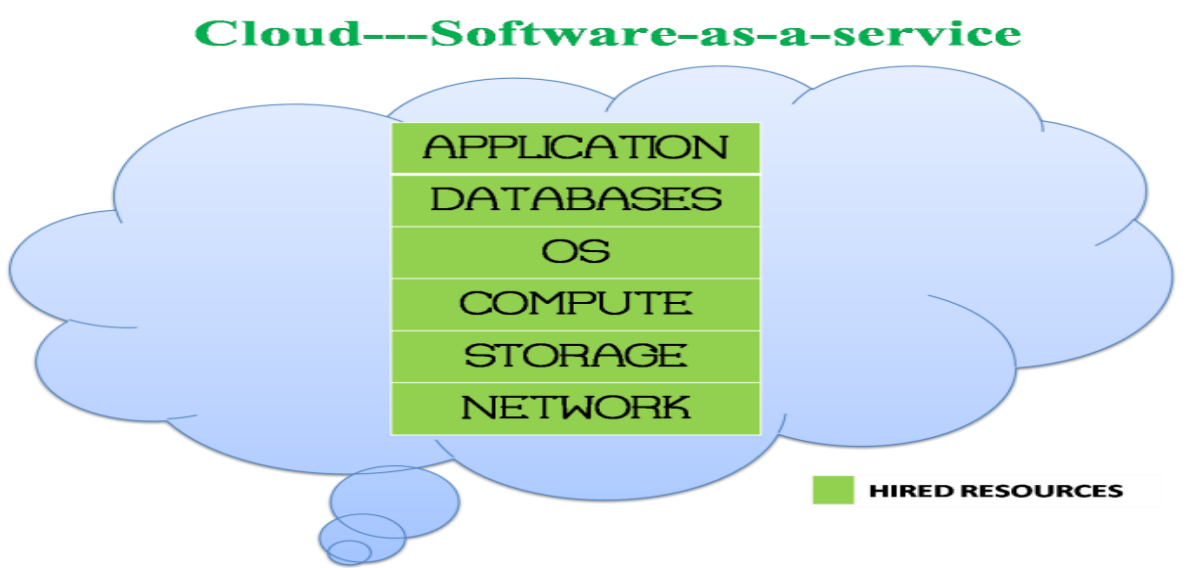

Cloud Deployment Models

\section{Cloud Deployment Model - Private Cloud}

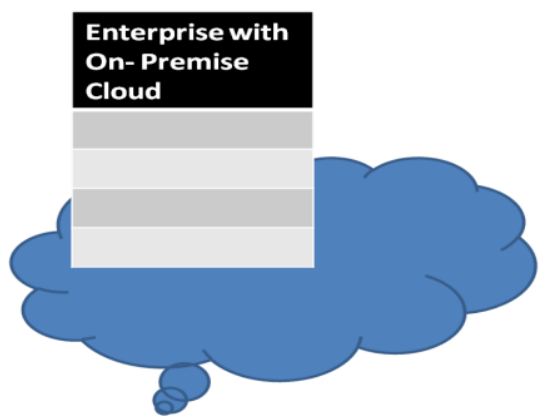

The key steps in deploying a private cloud infrastructure are to:

- Deploy private cloud infrastructure from the different fabric resources in the data center.

- Deploy compute fabric through bare metal operating system deployments of Hypervisors.

- Discover, classify, and allocate our storage fabric for private cloud use.

- Abstract our networking fabric for use in private cloud.

- Pull these fabric resources together and create a cluster for use as the underlying infrastructure for the private cloud.

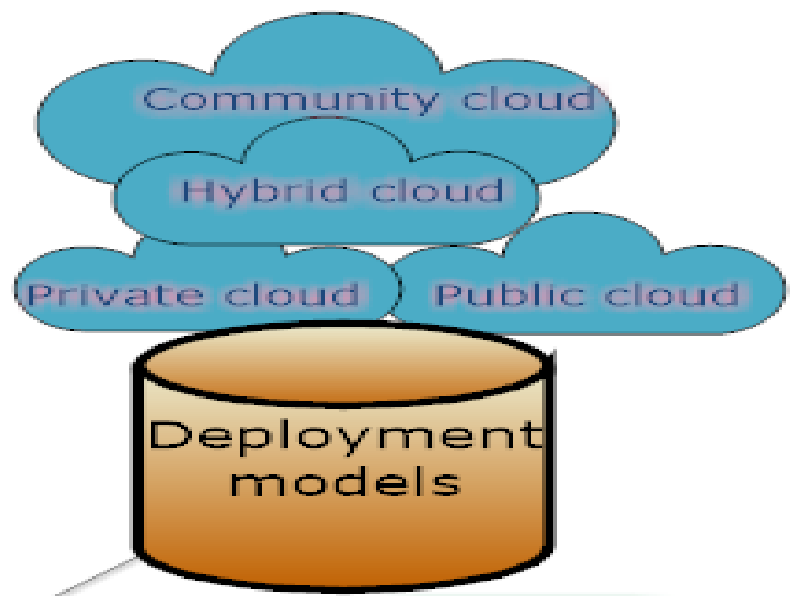

The cloud infrastructure consists of the following components:

- Physical Infrastructure which includes physical IT resources such as physical servers, storage systems and physical network components. Physical servers are connected to each other, to the storage systems, and to clients via physical networks.

- Virtual infrastructure which includes Resource pools (CPU, memory, network bandwidth, storage), Identity pools (vlan ID, mac address) and Virtual IT resources such as virtual machines, virtual volumes and virtual networks such as virtual switches and virtual NIC's. Virtual IT resources obtain capacity and identity from resource and identity pools. 
- Application and platform software such as OS, Database. These are created to provide software-as-aservice and Platform-as-a-service.

- Cloud Infrastructure Management and service creation tools consists of following components

- Virtual infrastructure management software - It creates pools of available physical infrastructure like creating storage pools, create vlan ID pools, CPU and memory pool. After creating pools this software help creating virtual infrastructure such as virtual machines, virtual LAN and virtual volumes.

- Unified management software - It interacts with virtual infrastructure software and collects information on existing physical and virtual infrastructure configurations. Unified software uses this information and provides a consolidated view of IT resources scattered across VDC's. This helps an administrator to monitor performance, capacity and availability of physical and virtual resources centrally. It also enables administrator to add capacity and identity to the existing pools. It passes configuration commands to the respective VDC management software and hence eliminates the administration of IT resources separately.

- User access management software - Allows users to request cloud services. It interacts with unified management software and forwards all service requests. It helps administrator to create and publish cloud service catalogue. It also authenticates users before fulfilling their service requests and monitors allocation or usage of resources associated with each Cloud service instance.

\section{ECOLOGICAL CONSEQUENCES OF Cloud COMPUTING}

Understanding the concepts and technologies of cloud computing will give us a broader idea of how it can affect the environment. Cloud computing can be understood as both and infrastructure and business model, where IT infrastructure, platform, software and data are delivered to you as a service via the Internet.

The two big giants of Information Technology business Microsoft and Google are good examples to explore the transformational consequence cloud computing is having on the industry and environment. As per the Microsoft "client-server" model of computing, each business buys its own servers and workstations, purchases expensive software licenses for everything from file servers to e-mail servers to office suite, and hires IT administration staff to keep everything running. For the last two and a half decades this has been an outrageously booming and money-spinning venture for Microsoft.

On the contrary, Google, who is one of the founders of contemporary cloud computing, promotes an entirely different model of computing. It does not advocate hosting e-mail servers, file servers and office suites on premise, running database servers, and purchasing numerous software licenses. With Google businesses simply use Google's merchandise - such as Gmail and Google Docs — through a web browser. According to one of the reports of Google around 2 million businesses have signed up for its cloud-based suite of products, and its espousal rate is only growing. The rapid use of Google's cloud-based services is now presenting a real warning to the Microsoft.

Cloud computing has helped greening our information technologies and labelled itself as an environmentally-friendly approach in delivering IT or computational power.

According to some quotes from industry regarding the effects of cloud computing technology, hypothetically we all agree that it helps in keeping environment greener even when consumption of IT and computational power is increasing everywhere and is becoming an important part of the lives of the people across the globe.

Reuven Cohen, CTO of Enomaly, a Cloud provider writes, "The general consensus says that reducing the number of hardware components and replacing them with remote cloud computing systems reduces energy costs for running hardware and cooling as well as reduces your carbon foot print while higher DC consolidation / optimization will conserve energy." Mr. Urquhart's way of thinking aligns with the same thought in a different way, "I believe one thing to be true: the increased efficiency of the hardware components in most cloud data centers and the increased utilization of these components mean that we are almost certainly doing more work per unit of energy consumed than before."

But as Mr. Cohen and James Urquhart (Product Marketing Manager, Cloud Computing and Virtualized Data Centres at Cisco) agree that there is no direct proof, no standard dimension or factor that everyone agrees indicates that the net effect is improved or poorer for the environment. And so for now we're really discussing the theoretical and using assumption to direct us in the right course.

We all can agree on one point that we are all getting more and more involved in using computation for almost all of our life works, hence usage of computer is increasing exponentially.

Imagine the computation used by millions of people who are connected through Face book or $\mathrm{g}$ talk and using other services of Google like YouTube for watching videos. This definitely requires a huge amount of data to be manipulated by the cloud infrastructures at various data centres that run numerous algorithms to deliver the experience and also balance data size, connection speed and client performance. Centralising all the computation and just delivering what a user requires over a thin client or mobile phone saves lot of infrastructure setup and finally waste. 
Greater effectiveness built into the hardware is a good thing for the environment - the lower the power consumed and the larger the amount of work done per clock tick of the processor is good for the environment.

\section{Cloud COMPUTING AS GREen COMPUTING}

In last decade Green computing is being discussed on a large scale, considering the effect of IT infrastructure on environment. Though information technology and environment are discussed separately, the ever-increasing impact of information technology on the environment can't be ignored. According to McKinsey report

- It is estimated that IT, considered as a whole, produces almost 1 gig ton of emissions a year, accounting for about 2 percent of total global emissions.

- By the year 2020 it is estimated to become about 1.54 gig tons or 3 percent of global emissions.

- Although the rising demand for computing cannot be controlled, we need to find out ways through which we can deliver computing in a more resourceful, ecologically welcoming way. Cloud computing technology can deliver such efficiencies but still as it is theoretical, giving rise to the expected query: Can shifting more of our computational load to the cloud help keep the environment safe and greener?

The changing Information Technology advancement trends towards cloud computing resembles the changing trends during the manufacturing uprising. Before Henry Ford introduced the concept to integrate and make more efficient assembly of vehicles, cars were pulled together one-by-one by craftsmen in expert shops. The beginning of the modern assembly line guided to a period of bulk-produced, well-built, and reasonably priced vehicles. Similarly different software and hardware are set up by different technology craftsmen whom we call as software engineers, hardware engineers or IT human resources. Now cloud computing advent also relies on concept of modern centralised industrial unit to provide Information Technology as a service. In the recent trends we have even advanced to concept of XAAS i.e. everything as a service in delivery of IT. Here these centralised factories in information technology are being established by Amazon, Google and Microsoft who produce and deliver everything as a service in IT.

The modernization that lies beneath these cloud computing factories is "virtualization technology." Before the development of virtualization, each server was used or dedicated to a given task. An e-mail server, for example, couldn't also act as a file server and as a database server because different server software's tended to meddle with each other and race for system resources. Hence to keep performance and reliability over efficiency the practice was to dedicate a server for a particular service.

In a conventional data centre where dedicated servers for particular services are used it is found that resources are idle most of the time yet they consume power. According to a McKinsey study server utilization is only 6 percent on an average and rest 94 percent of the time servers are sitting unused while they continue to consume considerable amounts of power. This entire unused and power consuming IT infrastructure leads to lot of IT waste and is responsible for carbon emission which poses danger to environment.

Virtualization enables many "virtual machines" to be run on a single physical server by abstracting the physical infrastructure. The individual virtual machines are entirely secluded from one another, providing the performance and reliability benefits of a committed server while using complete benefit of the computation resources with optimal utilisation of the host physical server. If one virtual machine stop working, the other virtual machines will remain totally unaffected.

IBM started the concept of virtualisation during 1960s but its potential has been gully used recently when conventional data centres were migrated and advanced to be virtualised data centre which is the first step in the journey of cloud computing set up. It has changed computing as a utility like electricity which can be provided on demand as a service.

In conventional data centres with dedicated servers in client-server computing model and with everdecreasing costs of servers has proliferated servers in businesses of all sizes. A report from market research firm IDC expected nearly 40 million servers in operation by 2011, up from 19 million in 2001 . With the help of virtualisation and cloud computing the computational needs of same 40 million servers could be met with a simple 2.4 million servers operating at 100 percent capacity. Virtualization enables servers to operate at levels nearer to their speculative upper limit. Cloud service providers automatically power down servers and resources that aren't needed to meet current demand levels.

Reports from HP, who have shifted their conventional data centres to virtualised data centre and finally into private cloud are able to consolidate their 85 data centres staffed by 19,000 IT workers to six cloud data centres with half number of IT employees.

\section{CONCLUSION}

Cloud computing is the most energy-efficient method for ever-increasing demand for IT services. Although the planning of cloud computing with a base on virtualization is much more efficient than traditional data centers, but claiming cloud computing as green computing will practically be possible if we are able to run 
these virtualized data centers with renewable sources of energy so as to reduce the overall ecological impact due to IT waste, power consumption and carbon emissions.

\section{REFERENCES}

[1] R. Buyya, High Performance Cluster Computing: Systems and Architectures, Prentice Hall, 1999.

[2] J. Yu and R. Buyya, "A taxonomy of scientific workflow systems for grid computing," ACM Sigmod Record, vol. 34, no. 3, 2005, pp. 44-49.

[3] M. Almorsy, et al., "Collaboration-Based Cloud Computing Security Management Framework," Proc. Cloud Computing (CLOUD), 2011 IEEE International Conference on, pp. 364-371.

[4] A. Behl and K. Behl, "An analysis of cloud computing security issues," Proc. Information and Communication Technologies (WICT), 2012 World Congress on, pp. 109-114.

[5] R. Buyya, et al., Cloud computing: principles and paradigms, Wiley, USA.

[6] S. Sengupta, et al., "Cloud Computing Security--Trends and Research Directions," Proc. Services (SERVICES), 2011 IEEE World Congress on, pp. 524-531.

[7] M. Hojabri and K.V. Rao, "Innovation in cloud computing: Implementation of Kerberos version5in cloud computing in order to enhance the security issues," Proc. Information Communication and Embedded Systems (ICICES), 2013 International Conference on, pp. 452-456.Y.T. Yu, M.F. Lau, "A comparison of MC/DC, MUMCUT and several other coverage criteria for logical decisions", Journal of Systems and Software, 2005, in press.

[8] Brian Dougherty, Jules White, and Douglas C. Schmidt. 2012. Model-driven auto-scaling of green cloud computing infrastructure. Future Gener. Comput. Syst. 28, 2 (February 2012), 371-378. DOI=10.1016/j.future.2011.05.009 http://dx.doi.org/10.1016/j.future.2011.05.009

[9] [1] M. Armbrust, A. Fox, R. Griffith, A. D. Joseph, R. Katz, A. Konwinski, G. Lee, D. Patterson, A. Rabkin, I. Stoica, and M. Zaharia, "Above the Clouds: A Berkeley View of Cloud Computing", EECS Department, University of California, Berkeley, Technical Report No., UCB/EECS-2009-28, pages 1-23, February 2009.

[10] R. W. Lucky, "Cloud computing”, IEEE Journal of Spectrum, Vol. 46, No. 5, May 2009, pages 27-45. M. D. Dikaiakos, G. Pallis, D. Katsa, P. Mehra, and A. Vakali, "Cloud Computing: Distributed Internet Computing for IT and Scientific Research", IEEE Journal of Internet Computing, Vol. 13, No. 5, September/October 2009, pages 10-13.

[11] G. Pallis, "Cloud Computing: The New Frontier of Internet Computing", IEEE Journal of Internet Computing, Vol. 14, No. 5, September/October 2010, pages 70-73.

[12] B. P. Rima, E. Choi, and I. Lumb, "A Taxonomy and Survey of Cloud Computing Systems", Proceedings of 5th IEEE International Joint Conference on INC, IMS and IDC, Seoul, Korea, August 2009, pages 44-51.

[13] R. Mata-Toledo, and P. Gupta, "Green data center: how green can we perform", Journal of Technology Research, Academic and Business Research Institute, Vol. 2, No. 1, May 2010, pages 1-8.

[14] S. K. Garg, C. S. Yeob, A. Anandasivamc, and R. Buyya, "Environment-conscious scheduling of HPC applications on IJCSI International Journal of Computer Science Issues, Vol. 9, Issue 1, No 1, January 2012 ISSN (Online): 1694-0814 www.IJCSI.org 244 Copyright (c) 2012 International Journal of Computer Science Issues. All Rights Reserved.

[15] http://www.northwestern.edu/newscenter/stories/2013/06/is-the-cloud-the-greenest-way-to-go.html 